\title{
A New Bandwidth Interval Based Forecasting Method for Enrollments Using Fuzzy Time Series
}

\author{
Hemant Kumar Pathak ${ }^{1}$, Prachi Singh ${ }^{2}$ \\ ${ }^{1}$ S. O. S. in Mathematics, Pandit Ravishankar Shukla University, Raipur, India \\ ${ }^{2}$ Government VYT Post Graduate Autonomous College, Durg, India \\ E-mail:hkpathak@sify.com,prachibksingh@gmail.com \\ Received February 25, 2011; revised March 11, 2011; accepted March 14, 2011
}

\begin{abstract}
In this paper, we introduce the concept of $(4 / 3) \sigma$ bandwidth interval based forecasting. The historical enrollments of the university of Alabama are used to illustrate the proposed method. In this paper we use the new simplified technique to find the fuzzy logical relations.
\end{abstract}

Keywords: Fuzzy Sets, Fuzzy Time Series, Fuzzy Logical Relations

\section{Introduction}

For planning the future forecasting plays an important role. During last few decades, various approaches have been developed for forecasting data of dynamic and nonlinear in nature. Fuzzy theory [1] has been successfully employed to prediction. Many studies on forecasting using fuzzy logic time series have been discussed such as enrollments, the stock index, temperature and fi- nancial forecasting. Some researchers used time invariant model and some used time variant model. The traditional statistical approaches can not predict problems in which the values are in linguistic terms.

After introduction of fuzzy sets by Zadeh [1], Song and Chissom [2] presented the definition of fuzzy time series and outlined its model by means of fuzzy relation equations, and approximate reasoning. They applied the model for forecasting under fuzzy environment in which historical data are of linguistic values. In that article, they showed that a universal forecasting method using fuzzy sets can be derived from the model of his process. After then many researchers ([2-7]) used this data to forecast. Cheng et al. [8] presented the trend-weighed fuzzy time model for TAIEX forecasting. Song et al. [2] and [9] used the relationship model, in which they constructed a relation matrix to relate the fuzzified enrollments of year $(i-1)$ and year $i$. Chen [3] presented a method which has the advantage of reducing the calculation time and simplifying the calculation process. Chen et al. [10] used the differences of the enrollments to present a method to forecast the enrollments of the University of Alabama.
Huang [11] extended Chen's [3] work and used simplified calculations with the addition of heu- ristic rules to forecast the enrollments. Chen [4] presented a forecasting method based on high-order fuzzy time series for forecasting the enrollments of the University of Alabama. Most of the forecasting methods require fuzzy relation. All such methods have following drawbacks:

1) Framing of fuzzy relation requires a lot of computations.

2) Computation cost is very high.

However, obtaining accurate forecast of student enrollment is not an easy task, as many factors determine the impact of the enrolment numbers. So, in the proposed method we introduced the interval based forecasting, which gives most plausible range of enrollments.

\section{Basic Concepts of Fuzzy Time Series}

Let $U=\left\{u_{1}, u_{2}, u_{3}, u_{4}, \cdots, u_{n}\right\}$ be the universe of discourse and let $A=\left|f_{A}\left(u_{1}\right) / u_{1}\right|+\left|f_{A}\left(u_{2}\right) / u_{2}+\cdots+\right| f_{A}\left(u_{n}\right) / u_{n} \mid$ be the fuzzy set defined on $U$. Here $f_{A}: U \rightarrow[0,1]$ is the membership function of $A, f_{A}\left(u_{i}\right), \forall i \in[1, n]$ indicates the grade of membership of $u_{i}$ in the fuzzy set $A$.

\subsection{Fuzzy Time Series}

Let $X(t)(t=0,1,2, \cdots)$ be the universe of discourse and the fuzzy set defined on $X(t)$ be $f_{i}(t)(t=0,1,2, \cdots)$. Then $F(t)=f_{i}(t) t=0,1,2, \cdots, i=1,2, \cdots$ the collection of all fuzzy sets defined on $X(t)$ is called a fuzzy time series of $X(t)(t=0,1,2, \cdots)$. 


\subsection{Fuzzy Relation}

If $F(t)$ is caused by $F(t-1)$, denoted by $F(t) \rightarrow F(t-1)$, then this relationship can be represented by $F(t)=F(t-1)$ * $R(t, t-1)$, where * denotes the composition operator and $R(t, t-1)$ is a fuzzy relation between $F(t)$ and $F(t-1)$.

\subsection{First Order Model}

The model in which the relation $R(t, t-1)$ is a fuzzy relation between $F(t)$ and $F(t-1)$ is called the first order model of $F(t)$.

\subsection{Time Invariant Fuzzy Time Series}

If in first order model of $F(t)$ relation $R(t, t-1)=R(t-1$, $t-2)$ for any time $t$, then $F(t)$ is called time invariant fuzzy time series.

\subsection{Time Variant Fuzzy Time Series}

If in first order model of $F(t)$ relation $R(t, t-1) \neq R(t-1$, $t-2$ ) for any time $t$, then $F(t)$ is called time invariant fuzzy time series.

\section{Proposed Method}

We now discuss our proposed method. The historical data and proposed method are shown in Table 1. Repeat Steps 1-3 of the method of Chen and Hsu [7] as follows. Step 1: Define the universe of discourse $U=[13000,20$ $000]$ and partition it into several even and length intervals $u_{1}=[13000,14000], u_{2}=[14000,15000], u_{3}=\left[\begin{array}{ll}15 & 000\end{array}\right.$, $16000], u_{4}=\left[\begin{array}{lll}16 & 000,17000\end{array}\right], u_{5}=\left[\begin{array}{lll}17 & 000,18000\end{array}\right]$,

Table 1. Historical data and proposed method.

\begin{tabular}{|c|c|c|c|c|c|}
\hline Year & Actual data & Fuzzified input & Fuzzified output & Calculated enrollmnt & Forecasted interval \\
\hline 1971 & 13055 & A1 & & & \\
\hline 1972 & 13563 & A2 & $\mathrm{A} 1$ & 13250 & [12 104, 14 396] \\
\hline 1973 & 13867 & $\mathrm{~A} 2$ & $\mathrm{~A} 2$ & 13750 & {$[12604,14896]$} \\
\hline 1974 & 14696 & A3 & $\mathrm{A} 2$ & 13750 & {$[12604,14896]$} \\
\hline 1975 & 15460 & A5 & $\mathrm{A} 3$ & 14500 & {$[13354,15646]$} \\
\hline 1976 & 15311 & A5 & A5 & 15375 & {$[14229,16521]$} \\
\hline 1977 & 15603 & A6 & A5 & 15375 & {$[14229,16521]$} \\
\hline 1978 & 15861 & A7 & A6 & 15625 & {$[14479,16771]$} \\
\hline 1979 & 16807 & A9 & A7 & 15875 & {$[14729,17$ 021] } \\
\hline 1980 & 16919 & A9 & A9 & 16833 & [15 687, 17 979] \\
\hline 1981 & 16388 & A8 & A9 & 16833 & [15 687, 17 979] \\
\hline 1982 & 15433 & A5 & A8 & 16500 & {$[15354,17646]$} \\
\hline 1983 & 15497 & A5 & $\mathrm{A} 5, \mathrm{~A} 6$ & 15500 & {$[14354,16$ 646] } \\
\hline 1984 & 15145 & A4 & $\mathrm{A} 5, \mathrm{~A} 6$ & 15500 & {$[14354,16646]$} \\
\hline 1985 & 15163 & A4 & A4 & 15125 & {$[13979,16$ 271] } \\
\hline 1986 & 15984 & A7 & A4 & 15125 & {$[13979,16$ 271] } \\
\hline 1987 & 16859 & A9 & A9 & 16833 & [15 687, 17 979] \\
\hline 1988 & 18150 & A 10 & A8, A9 & 16667 & {$[15521,17813]$} \\
\hline 1989 & 18970 & A11 & A 10 & 18125 & {$[16$ 979, 19 271] } \\
\hline 1990 & 19328 & A12 & A11 & 18750 & {$[17604,19896]$} \\
\hline 1991 & 19337 & A12 & $\mathrm{A} 12$ & 19500 & {$[18354,20646]$} \\
\hline 1992 & 18876 & A11 & A12 & 19500 & {$[18354,20646]$} \\
\hline
\end{tabular}


$u_{6}=[18000,19000], u_{7}=\left[\begin{array}{lll}19 & 000,20000\end{array}\right.$.

Sort the intervals based on the number of historical enrollment data in each interval from the highest to lowest and find the interval having largest number of data. Redivide this interval into four equal parts. Find the interval having second largest number data and re-divide it in three equal length sub-intervals find the interval having third largest number of data and re-divide it in two equal length sub-intervals. If there are no data in any interval then discard this interval. In this case the new distribution is shown in Table 2.

Step 2: Re-divide the intervals and rename them as follows: $u_{1}=$ [13 000, 13 500], $u_{2}=$ [13 500, 14 000], $u_{3}$ $=[14000,15000], u_{4}=\left[15000,15250, u_{5}=[15250,15\right.$ 500], $u_{6}=[15500,15750], u_{7}=[15750,16000], u_{8}=$ $\left[16333,16\right.$ 667], $u_{9}=[16667,17000], u_{10}=[18000,18$ $500], u_{11}=[18500,19000], u_{12}=$ [19 000, 20000$]$.

Step 3: Define each fuzzy set based on the re-divided intervals and fuzzify the data shown in Table 1, where fuzzy set $A i$ denotes a linguistic value of the data represented by a fuzzy set.

$$
\begin{aligned}
A 1 & =\text { very }^{4} \text { few }=1 / u_{1}+0.5 / u_{2} \\
A 2 & =\text { very }^{3} \text { few }=0.5 / u_{1}+1 / u_{2}+0.5 / u_{3} \\
A 3 & =\text { very }^{2} \text { few }=0.5 / u_{2}+1 / u_{3}+0.5 / u_{4} \\
A 4 & =\text { very few }=0.5 / u_{3}+1 / u_{4}+0.5 / u_{5} \\
A 5 & =\text { few }=0.5 / u_{4}+1 / u_{5}+0.5 / u_{6} \\
A 6 & =\text { moderate }=0.5 / u_{5}+1 / u_{6}+0.5 / u_{7} \\
A 7 & =\text { many }=0.5 / u_{6}+1 / u_{7}+0.5 / u_{8} \\
A 8 & =\text { very many }=0.5 / u_{7}+1 / u_{8}+0.5 / u_{9} \\
A 9 & =\text { too many }=0.5 / u_{8}+1 / u_{9}+0.5 / u_{10} \\
A 10 & =\text { too many }{ }^{2}=0.5 / u_{9}+1 / u_{10}+0.5 / u_{11} \\
A 11 & =\text { toomany }{ }^{3}=0.5 / u_{10}+1 / u_{11}+0.5 / u_{12} \\
A 12 & =\text { too many }{ }^{4}=0.5 / u_{11}+1 / u_{12}
\end{aligned}
$$

For simplicity the membership values of fuzzy set $A i$ are either $0,0.5,1$. Notice that we have not displayed the membership value 0 .

Now we give the steps of our proposed method.

Step 4: Fuzzify the data on Table 1. The reason for fuzzifying is to translate crisp values fuzzy sets to get a fuzzy time series. Now establish fuzzy logical relationships based on fuzzified data as " $A j \rightarrow A k$ " means if the fuzzified enrollments of year $(n-1)$ is $A j$ then the fuzzified enrollments of year $n$ is $A k$.

Step 5: By Table 3 it is clear that the fuzzy logical relationship groups are as follows.

Step 6: The fuzzified output is obtained by fuzzified input of previous years if 1) fuzzified input of $n$th year is $A i$ then fuzzified output of $(n+1)$ th year is also $A i$ (as in years $1971,1972, \cdots) .2$ ) If the fuzzified input of $n$th year
Table 2. Frequency of data.

\begin{tabular}{cc}
\hline Intervals & No. of data \\
\hline$[13000,14000]$ & 3 \\
{$[14000,15000]$} & 1 \\
{$[15000,16000]$} & 9 \\
{$[16000,17000]$} & 4 \\
{$[17000,18000]$} & 0 \\
{$[18000,19000]$} & 3 \\
{$[19000,20000]$} & 2 \\
\hline
\end{tabular}

Table 3. Logical groups.

\begin{tabular}{cc}
\hline Serial No. & Fuzzy logical relationship groups \\
\hline 1 & $A 1 \rightarrow A 2$ \\
2 & $A 2 \rightarrow A 2, A 2 \rightarrow A 3$ \\
3 & $A 3 \rightarrow A 5$ \\
4 & $A 4 \rightarrow A 4, A 4 \rightarrow A 7$ \\
5 & $A 4, A 5 \rightarrow A 5, A 5 \rightarrow A 6$ \\
6 & $A 7 \rightarrow A 7$ \\
7 & $A 8 \rightarrow A 5$ \\
8 & $A 9 \rightarrow A 9, A 9 \rightarrow A 8, A 9 \rightarrow A 10$ \\
9 & $A 10 \rightarrow A 11$ \\
10 & $A 11 \rightarrow A 12$ \\
11 & $A 12 \rightarrow A 11, A 12 \rightarrow A 12$ \\
12 &
\end{tabular}

is $A i$ and in previous years we have got more relations as $A i \rightarrow A j, A i \rightarrow A k, \cdots$ then the fuzzified output will be $(A j$, $A k, \cdots)$ (as in years $1983,1984,1988$ ).

Step 6: The fuzzified output is obtained by fuzzified input of previous years if 1)fuzzified input of $n$th year is $A i$ then fuzzified output of $(n+1)$ th year is also $A i$ (as in years $1971,1972, \cdots)$. 2)If the fuzzified input of $n$th year is $A i$ and in previous years we have got more relations as $A i \rightarrow A j, A i \rightarrow A k, \cdots$ then the fuzzified output will be $(A j$, $A k, \cdots)$ (as in years $1983,1984,1988$ ).

Step 7: Output values are the mid-values of the intervals in which the fuzzified output occurs.

Step 8: Next we calculate the mean, standard devia$\operatorname{tion}(\sigma)$ of output and interval by formula [output $-2 / 3 \sigma$, output $+2 / 3 \sigma]$.

Step 9: Now we can plot graphs of intervals lower limit of forecasted interval(LL of fore), upper limit of forecasted interval(UL of fore) and actual data to see that 


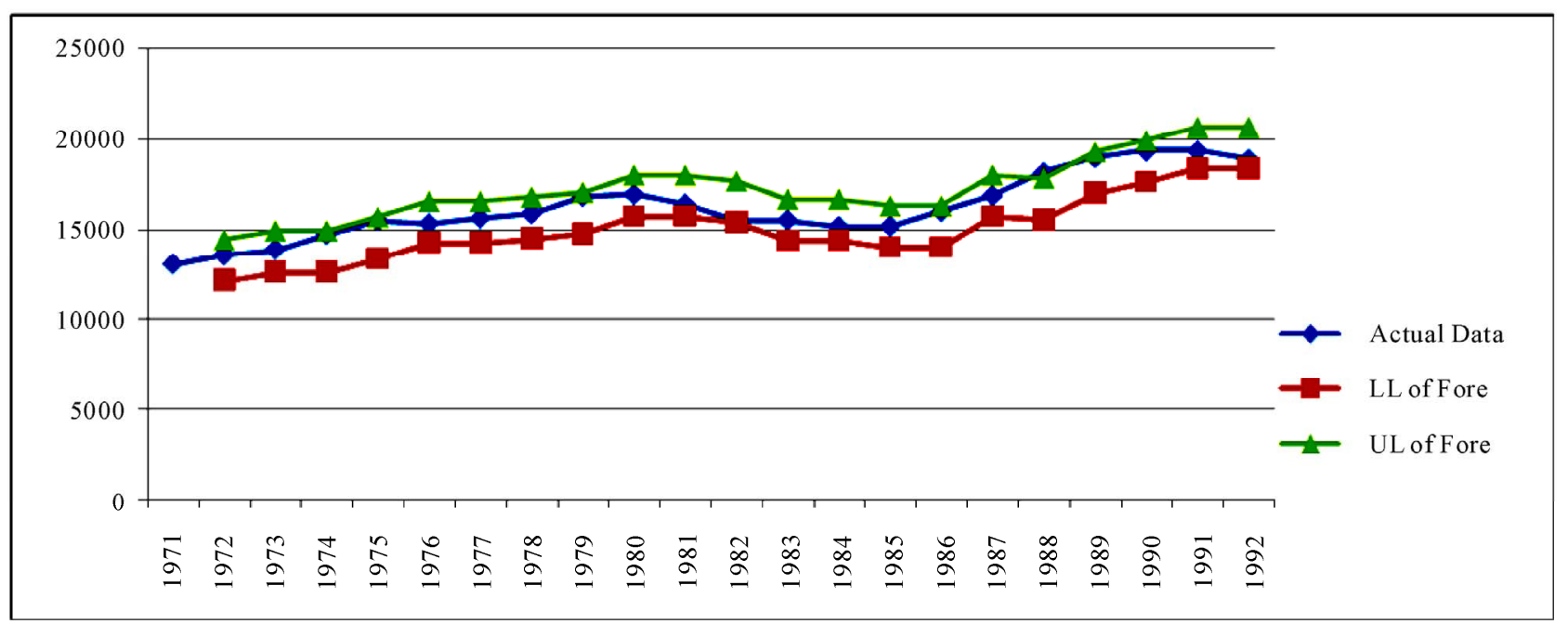

Figure 1. Graph of actual data and interval.

most of the actual data comes in the range of interval.

\section{Conclusions}

The development of technology and programming of languages with expert systems has considerably reduced the burden of decision makers. With regard to classical methods, fuzzy set theory give solutions in a quicker easier and most sensitive way.

In this proposed method there is no need of relationmatrix, so it reduces its calculation. It also reduces the next calculation for output by this relation-matrix.

The most remarkable thing in this method is that we give the most plausible range of forecasting, which is in the form of interval rather than a single value. It is also remarkable that in normal curve this interval is in the range $\pm 3 \sigma$ but in our method it is in the range of $\pm 2 / 3 \sigma$.

\section{References}

[1] L. A. Zadeh, "Fuzzy Sets," Information Control, Vol. 8, No. 3, 1965, pp. 338-353.

[2] Q. Song and B. S. Chissom, "Forecasting Enrollment with Fuzzy Time Series-Part I," Fuzzy Sets and Systems, Vol. 54, No. 1, 1993, pp. 1-9. doi:10.1016/0165-0114(93)90355-L

[3] S. M. Chen, "Forecasting Enrollments Based on Fuzzy Time Series," Fuzzy Sets and Systems, Vol. 81, No. 3, 1996, pp. 311-319. doi:10.1016/0165-0114(95)00220-0

[4] S. M. Chen, "Forecasting Enrollments Based on High
Order Fuzzy Time Series," Cybernetics and Systems: An International Journal, Vol. 133, No. 1, 2002, pp. 1-16.

[5] I. H. Kuo, S. J. Horng, T. W. Kao, C. L. Lee, T. L. Lin and Y. Pan, "An Improved Method for Forecasting Enrollment Based on Fuzzy Time Series and Particle Swarm Optimization," Expert Systems with Applications, Vol. 36, No. 3, 2009, pp. 311-319.

[6] S. M. Chen and C. C. Hsu, "A New Method to Forecast Enrollment Using Fuzzy Time Series," International Journal of Applied Science and Engineering, Vol. 3, No. 2, 2004, pp. 234-244.

[7] M. H. Lee, R. Efendi and Z. Ismail, "Modified Weighted for Enrollment Forecasting Based of Fuzzy Time Series," Matematika, Vol. 25, No. 1, 2009, pp. 67-78.

[8] C. H. Cheng, T. L. Chen and C. H. Chiang, "TrendWeighted Fuzzy Time Series Model for TAIEX Forecasting," Proceedings of the 13th International Conference on Neural Information Processing, Part-III, Lecture Notes in Computer Science, Hong Kong, Vol. 4234, 3-6 October 2006, pp. 469-477.

[9] Q. Song and B. S. Chissom, "Fuzzy Time Series and Its Models," Fuzzy Sets and Systems, Vol. 54, No. 3, 1993, pp. 267-277.doi:10.1016/0165-0114(93)90372-O

[10] S. M. Chen and J. R. Hwang, "Temperature Prediction Using Fuzzy Time Series," IEEE Transactions on Systems, Man and Cybernatics-Part B: Cybrnetics, Vol. 30, No. 2, 2000, pp. 263-275.

[11] K. Huarng, "Effective Lengths of Intervals to Improve Forecasting in Fuzzy Time Series," Fuzzy Sets and Systems, Vol. 123, No. 3, 2001, pp. 387-394. doi:10.1016/S0165-0114(00)00057-9 